A universal artificial intelligence platform for collaborative management of cataracts (see page 1553)

Universal AI-based multilevel platforms have the potential to make accurate cataract diagnoses and detect referral cases after evaluation of severity. The novel tertiary referral pattern achieved the anticipated population coverage (10.2 times greater than before) and referral rate.

Angiotensin converting enzyme inhibitor use and risk of cataract-a case-control analysis (see page 1561)

Contrary to a previous observational study suggesting an increased risk of cataract surgery after exposure to ACE inhibitors (ACEI), long-term ACEI use was not associated with a materially increased risk of cataract in our large observational study.

Dome-shaped macula: a potential protective factor for visual acuity after cataract surgery in patients with high myopia (see page 1566)

Dome-shaped macula was found in $13.8 \%$ of highly myopic eyes scheduled for cataract surgery. Associated with fewer visual threatening macular complications, it may be beneficial for visual function after cataract surgery in highly myopic eyes.

Areal summed corneal power shift is an important determinant for axial length elongation in myopic children treated with overnight orthokeratology (see page 1571)

Greater areal summed corneal power shift achieved at early period of orthokeratology treatment is associated with a long-term impact on reducing axial length elongation in myopic children.

Inner macular layer thickness by spectral domain optical coherence tomography in children and adults: a hospital-based study (see page 1576)

This retrospective study including 573 Korean subjects provides a normative range of each inner retinal layer thickness measured by SD-OCT. Inner sector mGCL thickness positively correlated with age, male gender and circumpapillary RNFL thickness.
Choroidal thickness and vascular density in macular telangiectasia type 2 using enface swept-source optical coherence tomography (see page 1584)

Utilising swept-source optical coherence tomography, the authors observed no statistically significant difference in choroidal thickness and choroidal vessel densities between eyes with Macular Telangiectasia Type two and control eyes after accounting for confounding variables.

Retinal microvascular abnormalities in neurofibromatosis type 1 (see page 1590) The current study identifies three different patterns of retinal microvascular abnormalities: simple vascular tortuosity, corkscrew retinal vessels and moya-moya-like arrangement, as typical manifestations of NF-1.

Classification of disease severity in retinitis pigmentosa (see page 1595)

In this cross-sectional study of 181 eyes of 93 patients with RP, the authors used visual acuity, visual field diameter, and ellipsoid zone width to classify disease severity from Grade 0 to 5 .

Early hydroxychloroquine retinopathy: optical coherence tomography

abnormalities preceding Humphrey visual field defects (see page 1600)

In this multicenter, retrospective case series, 17 eyes in 10 individuals with high-risk hydroxychloroquine intake exhibited early optical coherence tomography (OCT) abnormalities with normal Humphrey visual field testing, supporting a screening approach that utilises OCT.

Association between diabetic retinopathy and incident cognitive impairment (see page 1605)

Diabetic retinopathy, particularly in its advanced stages, is associated with an increased risk of developing cognitive impairment. Strategies to prevent diabetic retinopathy may reduce the risk of cognitive impairment onset in people with diabetes.

Estimation of impact of RPE65-mediated inherited retinal disease on quality of life and the potential benefits of gene therapy (see page 1610)

This study estimated substantial differences in quality of life weights (utilities) related to different levels of vision loss in RPE65-mediated inherited retinal disease. The data will support economic analyses of a new gene therapy.

\section{Optical coherence tomography} angiography findings in cystoid macular degeneration associated with central serous chorioretinopathy (see page 1615) Choroidal neovascularisation (CNV) is seen in the majority of patients with central serous chorioretinopathy and cystoid changes on optical coherence tomography angiography and should be suspected in the presence of a shallow neurosensory detachment.

Five-Year nationwide incidence of macular hole requiring surgery in Korea (see page 1619)

The incidence of macular hole requiring surgery from 2011 to 2015 in Korea was 3.14 (95\% CI, 3.07 to 3.21) per 100000 person-years in this largest nationwide population-based study of the incidence of macular hole.

Parameters affecting postoperative success of surgery for stage 4A/4B ROP (see page 1624)

The anatomical and functional success rate of vitreoretinal surgery was encouraging in stage 4 retinopathy of prematurity. The major factors affecting the success rate of the surgery were stage of the disease, preoperative treatment status, presence of preoperative plus disease, postoperative vitreous haemorrhage, induction of posterior hyaloid detachment, sparing the lens and iatrogenic tear formation.

Adalimumab for non-infectious uveitis: is it cost-effective? (see page 1633)

Adalimumab is clinically effective for treating non-infectious posterior segment uveitis. Its cost-effectiveness is uncertain due to scarcity of evidence but it appears to be more cost-effective in patients with active uveitis at high risk of blindness.

Dexamethasone implant for noninfectious uveitis: is it cost-effective? (see page 1639)

Dexamethasone implants in adult patients with non-infectious posterior segment-involving uveitis appeared cost-effective using generally accepted UK thresholds. However, there is 
substantial uncertainty around these results and further primary research is recommended.

Current ophthalmology practice patterns for syphilitic uveitis (see page 1645)

The International Ocular Syphilis Study Group reports that syphilitic uveitis is most often posterior, associated with secondary syphilis, diagnosed serologically and treated with penicillin. Serologic testing of all patients with uveitis ensures timely identification.

18F-Fluorodeoxyglucose positron emission tomography is useful for the diagnosis of intraocular sarcoidosis in patients with a normal computerized tomography scan (see page 1650)

In almost $30 \%$ of the patients presenting with uveitis, there are positive findings on the 18F-FDG PET/CT consistent with sarcoidosis despite a normal chest CT.18F-FDG PET/CT should be considered as second line exam in selected patients.
Aurora borealis and string of pearls in vitreoretinal lymphoma: patterns of vitreous haze (see page 1656) Different pattern of vitreous haze can be associated with vitreoretinal lymphoma and their recognition on clinical examination and multimodal imaging may result in early suspicion of this disease. These patterns may vary in appearance, such as aurora borealis and string of pearls.

Refractory follicular conjunctival lesions: overlook as just inflammation or not? (see page 1660)

Conjunctival MALT lymphoma can present in various ways, not only with a salmon-patch appearance. Biopsy should be considered especially in cases of follicular lesions responding poorly to topical steroids.

Association of visual acuity with educational outcomes: a prospective cohort study (see page 1666)

In this school-based cohort study, better baseline presenting visual acuity, but not refractive error or spectacle wear, was a significant predictor of later academic performance, suggesting a significant association between vision and academic outcomes.

Utility of clock chart binocular edition for self-checking the binocular visual field in patients with glaucoma (see page 1672)

We developed a simple self-check chart (CLOCK CHART Binocular Edition) that helps patients with glaucoma notice abnormalities in the binocular VF and evaluated its usefulness.

Diagnostic performance of optical coherence tomography angiography in glaucoma: a systematic review and metaanalysis (see page 1677)

We provide a systematic review with meta-analysis of OCT-angiography diagnostic abilities in glaucoma, with pooled peripapillary vessel density significantly lower in glaucomatous eyes, among other diagnostic outcomes, comprising 1054 glaucomatous and 689 control eyes. 